\title{
Control and optimization with differential-algebraic constraints
}

\author{
L. Biegler (Carnegie Mellon Univ.) \\ S. L. Campbell (North Carolina State Univ.), \\ V. Mehrmann (TU Berlin)
}

October 24, 2010-October 29, 2010

\section{Overview of the Field}

Differential Algebraic Equations (DAEs) are mixed systems of differential and algebraic equations. It has been recognized for some time now that they have great potential both theoretically and in applications. DAEs form one of the most elegant and simple ways to model a physical system because they allow for the creation of separate models for subcomponents that can then be pasted together via a network. As a consequence, this concept is used in many modern CAD/modeling systems like SIMULINK, Scicos and DYMOLA, although most software packages cannot fully exploit the full potential of DAE models.

But this nice feature of DAEs for modeling has also a disadvantage, since it shifts all of the difficulties of a system onto the analysis and the numerical methods. For this reason in recent years much effort has been spent to analyze general DAEs and to derive suitable numerical methods either for general DAEs, see e.g. [9, 19, 18, 20, 29, 38] or for special DAEs arising in applications, see e.g. [6, 12, 28, 37].

This analytical and numerical work so far has been primarily driven by the simulation community, where the desire was to simulate the behavior of a complex system which could be electrical, mechanical, chemical, or all three. Due to the ever growing complexity of models which pose new challenges, the field is developing rather rapidly including now also hybrid [2, 3, 4, 11, 21, 31, 39] and delay systems [16, 17, 25, 26, 27].

Once a system can be modeled and simulated, there arises the need to control the process or optimize its performance. The control of physical processes is an important task in many applications and over the last two decades there have been tremendous advances in the theory and applications of control in almost all disciplines of science and technologies. Note that this includes not only the obvious applications such as designing a more efficient process, but also determining what are the control mechanisms inherent in complex biological systems and fitting models to data.

\section{Recent Developments and Open Problems}

As the two topics, simulation of DAEs and control/optimization, have evolved in recent years, there has been a growing awareness of interconnections which arise in a number of ways. One obvious way is the control of DAE modeled systems. Optimality conditions and a maximum principle for general DAEs have only recently been obtained [30] and the results are far from complete. But there are other more subtle connections in that the necessary conditions for an optimal control problem typically form a DAE and the solution of some control problems in the presence of constraints or invariants also involve DAEs. An open problem is in 
particular the analysis and numerical solution of the resulting optimality system and its proper regularization in the case of singular control.

Another important topic in which recently breakthroughs have been made is the stability analysis for DAEs, including the computation of Lyapunov exponents and Sacker-Sell spectra [32, 33]. Here an open problem is the extension of these results to fully nonlinear systems as well as the development of improved computational methods for large scale problems.

It is clear that another limiting factor is the growing size of DAE problems in application, ranging from several thousands in chemical engineering to billions of equations in circuit simulation. So in order to apply control and optimization methods, model reduction becomes an essential issue. However, classical approaches for model reduction lead to enormous difficulties when considering DAEs. This is mainly due to the fact that the constraints and physical properties, like stability and passivity, must be respected in the approximated model to achieve physically meaningful results $[1,35]$. On the other hand the approximated model must be efficiently computable. To resolve this conflict is an open problem in many areas, which can only be resolved by efficiently exploiting the structure or by making compromises.

Another recent connection between DAEs and control are hybrid systems of DAEs, where different DAE models are chosen depending on some switching function. Important applications are chemical engineering systems, mechanical systems with friction, or electronic circuits, where depending on the frequency different circuits are used in the simulation and control $[4,5]$.

In addition, there are strong synergies on the proper modeling and formulation of DAE-constrained optimization problems and their solution with recently developed algorithms for nonlinear programming and mixed integer nonlinear programming.

Due to the many connections, some researchers in the numerical DAE community have begun to consider control/optimization issues while at the same time some from the control/optimization community have begun to examine DAE issues [7, 8, 10, 13, 14, 15, 22, 23, 34, 36].

With these new developments and more and more applications emerging, and with the increasing complexity of problems it was absolutely essential to bring the different communities working in these areas together. In spite of this natural interest on both sides, this was the first workshop ever devoted to control/optimization and DAEs. It brought together the widely scattered researchers from a number of disciplines in academia and from industry, and we believe that is was a milestone in the move to the next major advances to occur in this fundamentally important field. There were participants from chemical engineering, aerospace engineering, and electrical engineering. It is absolutely clear that further advances require the intensive interdisciplinary cooperation for the solution of fundamental mathematical, numerical, and algorithmic concerns and to attack the challenges that arise in many applications.

\section{Presentation Highlights}

The workshop featured 30 one-hour talks and it was decided that each speaker should make a strong effort to integrate the different (numerical analysis, control, optimization and engineering) communities in the discussion. In most talks this was really achieved, and was documented by the intensive discussion during and after each talk. Almost all talks were of extremely high quality.

It would be difficult to list all the highlights during the week, so we just mention a few. The chosen examples represent several of the key application areas of optimization of DAE based systems, namely mechanics, chemical engineering, and electrical systems.

Thanos Antoulas presented a concept of generating system models in a very simple way by not insisting that the resulting model is a regular model. This again is an interesting approach which allows simpler modeling put puts a large burden on the analytic and numerical treatment afterwards. This approach fits in well with the DAE modeling philosophy of working with relationship models. Immediate discussions started right after the talk how to integrate current methods for staircase computation with this new modeling approach.

Roland Freund presented a new approach for model reduction for super-large DAEs arising in circuit simulation. He emphasized that it is essential to guarantee that system properties like stability and passivity must be preserved. He then presented a new method, which is proven to achieve this goal without sacrificing the approximation accuracy. Model reduction is important any time large models are involved. 
Francisco Borelli discussed new approaches to model predictive control. He, in particular, presented the need for new methods to control in real-time complex systems. As an example he used driver support systems in cars and trucks which support the interaction of the human driver, the vehicle and the environment. Another example concerned the optimal heating/cooling of buildings. He then suggested new approaches using robust control invariant sets and showed how these can be used efficiently.

Paul Barton, motivated by large complex problems in chemical engineering, discussed approaches for global optimization techniques with differential-algebraic constraints. He suggested a new approach for the relaxation and convexification of DAE constraints and how to put the constraints into interval boundaries. He demonstrated the approach for several applications using interval Newton-type methods.

Peter Kunkel presented a global optimality result for general nonlinear DAE optimal control problems and suggested several state-or-the-art developments. In particular he showed that the standard naive approach may lead to wrong results. He showed that it is possible to remodel the current model in what is called a strangeness-free formulation, that allows one to apply classical results for constrained optimization of optimal control problems with DAE constraints.

Serkan Gugercin showed that interpolation based model reduction methods can be constructed that directly work on higher order models or parametric models. The features of the new approach were demonstrated via several examples and it was shown that this new approach is very flexible towards different properties of the system.

Matthias Gerdts presented new results on local and global minimum principles for optimal control problems with mechanical DAE constraints. He demonstrated the quality of the results and their implementation via several examples from multibody dynamics.

\section{Scientific Progress Made}

Several research collaborations were started during the meeting and are continued afterwards. During every break and every night, several groups of people met for intense discussions, to work on new research projects or to discuss the talks of the previous sessions.

Many new collaborations were started and existing cooperations were refreshed.

To name a few examples, Tatjana Stykel and Serkan Gugercin worked almost every evening on new developments for model reduction of higher order and parametric systems.

Steve Campbell, Peter Kunkel, and Volker Mehrmann were able to carry out initial discussions on an examination of general regularization procedures for optimal control methods for general DAEs. Peter Kunkel's presentation served as the starting point for their discussions. Plans were made for further meetings over the next year and the framework for a paper giving a new general approach synthesizing several ideas presented at the workshop was developed.

Steve Campbell and John Betts had worked before. At the workshop a new collaboration was begun with Karmethia Thompson on direct transcription optimal control software for DAEs with delays. Their discussions at the workshop were especially stimulated by discussions with Larry Biegler about alternative collocation schemes for control problems and the handling of discontinuities which can occur with delays, with Bill Hager on the convergence of discretizations on optimal control problems solved by direct transcription, with Ray Spiteri who has used direct transcription software, and with Enright who has developed a number of delay numerical solvers. Ray Spiteri may also become involved in this collaboration.

Motivated by excitement generated at the workshop, the three organizers Larry Biegler, Steve Campbell, and Volker Mehrmann began a collaborative effort to produce a volume based on the workshop. This volume will be more than just a collection of talks but will also include a major introductory survey that the organizers will write. Initial reviews have been very favorable to the proposal. Both publishers that were given the proposal have expressed strong interest and a book contract is expected by early February. This volume is discussed more fully in the next section.

\section{Outcome of the Meeting}

The meeting was very intense and the efforts made by the speakers to integrate the different communities led to intensive discussions and cooperations. 
With the intense interaction between the different groups, it was felt that a research monograph with surveys on the state-of-the-art of the research in this field would be an essential accelerator for future research work. Too often, new results appear as focused research publications in specialized journals and do not get to other disciplines. Instead, to reach a wider audience, the participants decided to produce a monograph by summarizing selected topics from the workshop and making them accessible to the different communities. Most of the speakers have volunteered to write survey articles of an expository nature, that provide essential review material but especially also present new research directions and challenges. The volume will also include a major introductory survey by the organizers.

All the talks presented are publically available at the conference website.

\section{References}

[1] A. C. Antoulas, Approximation of large-scale dynamical systems, Society for Industrial and Applied Mathematics, Philadelphia, PA, 2005.

[2] A.P. Aguiar and A.M. Pascoal, From local to global stabilization: A hybrid system approach, CONTROLO2002, 5th Portuguese conference on automatic control, 2002.

[3] J. Agrawal, K.M. Moudgalya, and A.K. Pani, Sliding motion of discontinuous dynamical systems described by semi-implicit index one differential algebraic equations, Chemical Engineering Science, 61 (2006), 4722-4731.

[4] P.I. Barton and C.K. Lee, Modeling, Simulation, Sensitivity Analysis, and Optimization of Hybrid Systems, ACM Transactions on Modeling and Computer Simulation, 12 (2002), 256-289.

[5] P.I. Barton, C.K. Lee, and M. Yunt, Optimization of hybrid systems, Computers Chem. Engng., 30 (2006), 1576-1589.

[6] J.T. Betts, Practical Methods for Optimal Control using Nonlinear Programming, SIAM, Philadelphia, 2001.

[7] J.T. Betts and W.P. Huffman, Estimating adjoint variables from a direct transcription solution, M\&CT=TECH-04-001, Technical Document Series, Phantom Works, Mathematical and Computing Technology, Seattle, WA., 2004.

[8] J.T. Betts, S.L. Campbell, and A. Engelsone, Direct transcription solution of optimal control problems with higher order state constraints: theory vs practice, Optimization and Engineering, 8 (2007), 1-19.

[9] K.E. Brenan, S.L. Campbell, and L.R. Petzold, Numerical Solution of Initial-Value Problems in Differential-Algebraic Equations, SIAM Publications, 1996.

[10] S.L. Campbell and R. Marz, Direct transcription solution of high index optimal control problems and regular Euler-Lagrange equations, J. Comp. Appl. Math., 202, (2007), pp. 186-202.

[11] D. Chen, Stabilization of planar switched systems, Systems and Control Letters, 51 (2004), 79-88.

[12] E. Eich-Soellner and C. Führer. Numerical Methods in Multibody Systems, Teubner Verlag, Stuttgart, Germany, 1998.

[13] A.E. Englesone, Direct Transcription Methods in Optimal Control: Theory and Practice, PhD Thesis, Operations Research Program, North Carolina State University, 2006.

[14] A. Engelsone, S.L. Campbell, and J.T. Betts, Direct transcription solution of higher-index optimal control problems and the virtual index, Appl. Numerical Mathematics, 57 (2007), 281-196.

[15] A. Engelsone and S.L. Campbell, Adjoint estimation using direct transcription multipliers: compressed trapezoidal method, Optimization and Engineering, 9 (2008), 291-305. 
[16] E. Fridman, and U. Shaked, $H_{\infty}$ control of linear state-delay descriptor systems: an LMI approach, Linear Algebra Appl., 351/352 (2002), 271-302.

[17] E. Fridman, and U. Shaked, Delay-dependend $H_{\infty}$ control of uncertain discrete delay systems, Europ. J. Control, 11 (2005), 29-37.

[18] E. Griepentrog and R. März. Differential-Algebraic Equations and their Numerical Treatment. Teubner Verlag, Leipzig, Germany, 1986.

[19] E. Hairer, C. Lubich, and M. Roche, The Numerical Solution of Differential-algebraic Systems by Runge-Kutta Methods, Springer Lecture Notes in Mathematics No. 1409, 1989.

[20] E. Hairer and G. Wanner, Solving Ordinary Differential Equations II: Stiff and Differential-Algebraic Problems, Springer-Verlag, 1996.

[21] P. Hamann and V. Mehrmann, Numerical solution of hybrid systems of differential-algebraic equations, Comp. Meth. App. Mech. Eng., 197 (2008), 693-705.

[22] S. Kameswaran and L.T. Biegler, Simultaneous dynamic optimization strategies: recent advances and challenges, Computers and Chemical Engineering, 30 (2006), 1560-1575.

[23] S. Kameswaran and L.T. Biegler, Convergence Rates for Direct Transcription of Optimal Control Problems Using Collocation at Radau Points, Computational Optimization and Applications, 41, 1, pp. 81-126 (2008)

[24] Kameswaran, S. and L. T. Biegler, "Advantages of a Nonlinear Programming Based Methodologies for Inequality Path Constrained Optimal Control Problems - A Numerical Study," SIAM J. Scientific Computing, 30(2), pp. 957-981 (2008)

[25] V.L. Kharitonov and D. Hinrichsen, Exponential estimates for time delay systems, Technical Report 03-05, Zentrum f. Technomathematik, Univ. Bremen, 2005.

[26] V.L. Kharitonov and E. Plischke, Lyapunov matrices for time-delay systems, Technical Report 04-05, Zentrum f. Technomathematik, Univ. Bremen, 2005.

[27] V.L. Kharitonov and A.P. Zhabko, Lyapunov-Krasovskij approach to the robust stability analysis of time delay systems, Math. Control, Signals, Sys., 3 (1990), 211-224.

[28] A. Kumar and P. Daoutidis, Control of nonlinear differential-algebraic equation systems, CRC Press, 1999.

[29] P. Kunkel and V. Mehrmann, Differential-Algebraic Equations. Analysis and Numerical Solution, EMS Publishing House, Zürich, Switzerland, 2006.

[30] P. Kunkel and V. Mehrmann, Optimal control for unstructured nonlinear differential-algebraic equations of arbitrary index, MATHEMATICS OF CONTROL SignALS AND Systems, Vol. 20, 227-269, 2008.

[31] C. Lacoursière, Ghosts and Machines: Regularized Variational Methods for Interactive Simulation of Multibodies with Dry Frictional Contacts, PhD thesis, Umeå University, Sweden, 2007.

[32] V. H. Linh and V. Mehrmann. Lyapunov, Bohl and Sacker-Sell Spectral Intervals for DifferentialAlgebraic Equations. Journal on Dynamics And Differential Equations, Vol. 21, 153-194, 2009.

[33] V.H. Linh, V. Mehrmann, and E. Van Vleck, $Q R$ methods and Error Analysis for Computing Lyapunov and Sacker-Sell Spectral Intervals for Linear Differential-Algebraic Equations. Has appeared electronically in ADVANCES IN COMPUTATIONAL MATHEMATICS, 2010, DOI: $10.1007 / \mathrm{s} 10444-010-9156-1$.

[34] G. Mathew, I. Mezic, S. Grivopoulos, U. Vaidya, and L. Petzold, Optimal control of mixing in stokes fluid flows, J. Fluid Mech., 580 (2007), 261-281. 
[35] V. Mehrmann and T. Stykel. Balanced truncation model reduction for large-scale systems in descriptor form. In Dimension Reduction of LARGe-Scale Systems, P. Benner, V. Mehrmann and D. Sorensen, Edtrs., LNCSE Vol. 45. Springer Verlag, Heidelberg, 2005, pp. 83-115.

[36] L. Petzold, S. Li, Y. Cao, and R. Serban, Sensitivity analysis of differential-algebraic equations and partial differential equations, Computers and Chemical Engineering, 30 (2006), 1553-1559.

[37] P. J. Rabier and W. C. Rheinboldt, Nonholonomic Motion of Rigid Mechanical Systems from a DAE Viewpoint, SIAM Publications, Philadelphia, PA, 2000.

[38] P. J. Rabier and W. C. Rheinboldt. Theoretical and Numerical Analysis of Differential-Algebraic Equations, volume VIII of Handbook of Numerical Analysis. Elsevier Publications, Amsterdam, The Netherlands, 2002.

[39] L. Wunderlich, Analysis and Numerical Solution of Structured and Switched Differential-Algebraic Systems, PhD thesis, TU Berlin, 2008. 